\title{
Hands-on defibrillation and electrocardiogram artefact filtering technology increases chest compression fraction and decreases peri-shock pause duration in a simulation model of cardiac arrest
}

\author{
Shannon M. Fernando, MD, MSc ${ }^{*}{ }^{\dagger}$; Sheldon Cheskes, $\mathrm{MD}^{\ddagger}$; Daniel Howes, MD*\$
}

\section{ABSTRACT}

Background: Reducing pauses during cardiopulmonary resuscitation (CPR) compressions result in better outcomes in cardiac arrest. Artefact filtering technology (AFT) gives rescuers the opportunity to visualize the underlying electrocardiogram (ECG) rhythm during chest compressions, and reduces the pauses that occur before and after delivering a shock. We conducted a simulation study to measure the reduction of peri-shock pause and impact on chest compression fraction (CCF) through AFT. Methods: In a simulator setting, participants were given a standardized cardiac arrest scenario and were randomly assigned to perform CPR/defibrillation using the protocol from one of three experimental arms: 1) Standard of Care (pauses for rhythm analysis and shock delivery); 2) AFT (no pauses for rhythm analysis, but a pause for defibrillation); or 3) AFT with hands-on defibrillation (no pauses for rhythm analysis or defibrillation). The primary outcomes were CCF and peri-shock pause duration, with secondary outcomes of pre- and post-shock pause duration.

Results: AFT with hands-on defibrillation was found to have the highest CCF (86.4\%), as compared to AFT alone $(83.8 \%, p<0.001)$, and both groups significantly improved CCF in comparison with the Standard of Care $(76.7 \%, p<0.001)$. AFT with hands-on defibrillation was associated with a reduced peri-shock pause (2.6 seconds) as compared to AFT alone (5.3 seconds, $p<0.001$ ), and the Standard of Care (7.4 seconds, $p<0.001$ ).

Conclusions: In this cardiac arrest model, AFT results in a greater CCF by reducing peri-shock pause duration. There is also a small but detectable improvement in CCF with the addition of hands-on defibrillation.

\section{RÉSUMÉ}

Contexte: La diminution du nombre de pauses durant les manœuvres de réanimation cardiopulmonaire $(R C P)$ donne de meilleurs résultats dans le contexte de l'arrêt cardiaque. La technique de filtrage des parasites (TFP) permet aux secouristes de visualiser, à l'ECG, le rythme cardiaque sous-jacent durant les compressions thoraciques, et réduit ainsi le nombre de pauses avant et après les décharges électriques. L'étude en simulation visait à mesurer la réduction des pauses qui précèdent et suivent les chocs ainsi que leur incidence sur la fraction des compressions thoraciques (FCT), et ce, à l'aide de la TFP.

Méthode: Les participants à l'étude ont été placés, dans un contexte de simulation, devant un scénario uniforme d'arrêt cardiaque, puis on leur a demandé, après répartition au hasard, de procéder à la RCP et à la défibrillation selon I'un des trois protocoles expérimentaux suivants: 1) les " soins habituels " (pauses pour l'analyse du rythme et pauses pour les chocs); 2) la TFP seule (pas de pause pour l'analyse du rythme mais pause pour la défibrillation); 3) la TFP avec défibrillation et compressions thoraciques continues (CTC) (pas de pause pour l'analyse du rythme ni pour la défibrillation). Les principaux critères d'évaluation étaient la FCT et la durée des pauses précédant et suivant les chocs, et les critères secondaires d'évaluation consistaient en la mesure de la durée des pauses avant et après les chocs.

Résultats: La TFP alliée à la défibrillation et aux CTC a produit une FCT plus élevée $(86,4 \%)$ que la TFP seule $(83,8 \%$; $p<0,001)$, et dans les deux groupes il y a eu une amélioration importante de la FCT comparativement aux soins habituels $(76,7 \% ; p<0,001)$. En outre, la TFP alliée à la défibrillation et aux CTC a été associée à une diminution des pauses précédant et suivant les chocs $(2,6 \mathrm{~s})$ par rapport à la TFP seule $(5,3 \mathrm{~s} ; p<0,001)$ et aux soins habituels $(7,4 \mathrm{~s}, p<0,001)$. Conclusions: Dans le modèle d'arrêt cardiaque décrit ici, la TFP a permis d'augmenter la FCT par une diminution de la durée des pauses qui précèdent et suivent les chocs. De plus, I'association des CTC et de la défibrillation s'est traduite par une amélioration certes faible mais détectable de la FCT.

Keywords: cardiopulmonary resuscitation, cardiac arrest, defibrillation, chest compression fraction, perishock pause

From the *Kingston Resuscitation Institute, Kingston, ON; †Department of Emergency Medicine, University of Ottawa, Ottawa, ON; $¥$ Sunnybrook Centre for Prehospital Medicine, Department of Family and Community Medicine, Division of Emergency Medicine, University of Toronto, Toronto, ON; and §Departments of Emergency Medicine and Critical Care, Queen's University, Kingston, ON.

Correspondence to: Dr. Daniel Howes, Department of Emergency Medicine, Queen's University, Kingston General Hospital, 72 Stuart St, Kingston, ON K7L 2V6; Email: howesd@kgh.kari.net 


\section{INTRODUCTION}

Pauses in chest compressions during cardiopulmonary resuscitation (CPR) result in the reduction of perfusion pressure to vital organs. ${ }^{1,2}$ Guidelines have emphasized minimizing CPR pauses with the intent of maintaining coronary perfusion pressure and increasing the likelihood of return of spontaneous circulation. ${ }^{3,4}$ Previous work has demonstrated an association between increased chest compression fraction (CCF: the percentage of time during cardiac arrest that is spent performing chest compressions) and increased likelihood of discharge from the hospital in out-of-hospital cardiac arrest (OHCA). ${ }^{5}$ In particular, reduction in the peri-shock pause (the pause in chest compressions immediately before and after defibrillatory shock) has recently been shown to be associated with improved survival in OHCA. $^{6,7}$

Several pauses occur when CPR is performed according to current guidelines: CPR is paused when the patient is being ventilated with a mask, during rhythm analysis (due to motion artefact during chest compressions [Figure 1, A and B]), and when defibrillation is being performed.

It may be possible to further reduce the pauses in CPR. Recent advances in technology allow rhythm assessments to be accomplished during ongoing chest compressions. Artefact filtering technology (AFT) removes the artefact caused by chest compressions from the electrocardiogram (ECG) trace. ${ }^{8} \mathrm{AFT}$ is an adaptive filter that uses the sternal velocity signal from an accelerometer in a pad under the hands of the rescuer. This allows rescuers to see the underlying cardiac rhythm during chest compressions (see Figure 1C), negating the need to pause for rhythm analysis. Previous manikin-based research on this modality has demonstrated as much as an $80 \%$ reduction in preshock pause time through the use of AFT. ${ }^{9}$ This study did not use the standard advanced-cardiovascular-lifesupport (ACLS) algorithm (e.g., compressions were paused during defibrillator charging) and also employed a novice group of rescuers certified in basic life support only. The impact of AFT on the peri-shock pause has not been evaluated.

Another opportunity to minimize peri-shock pause is by reducing the interruption in compressions immediately prior to the delivery of energy during defibrillation. This pause is necessitated by the harm that may be caused to the rescuer by maintaining contact with the patient during defibrillation. This notion of rescuer safety has come under significant scrutiny in the recent literature, ${ }^{10}$ but it may be safe for a rescuer to continue compressions during energy delivery. ${ }^{11}$ The use of hands-on defibrillation (i.e., the continuation of chest compressions during delivery of a shock) has been proposed as a method of further
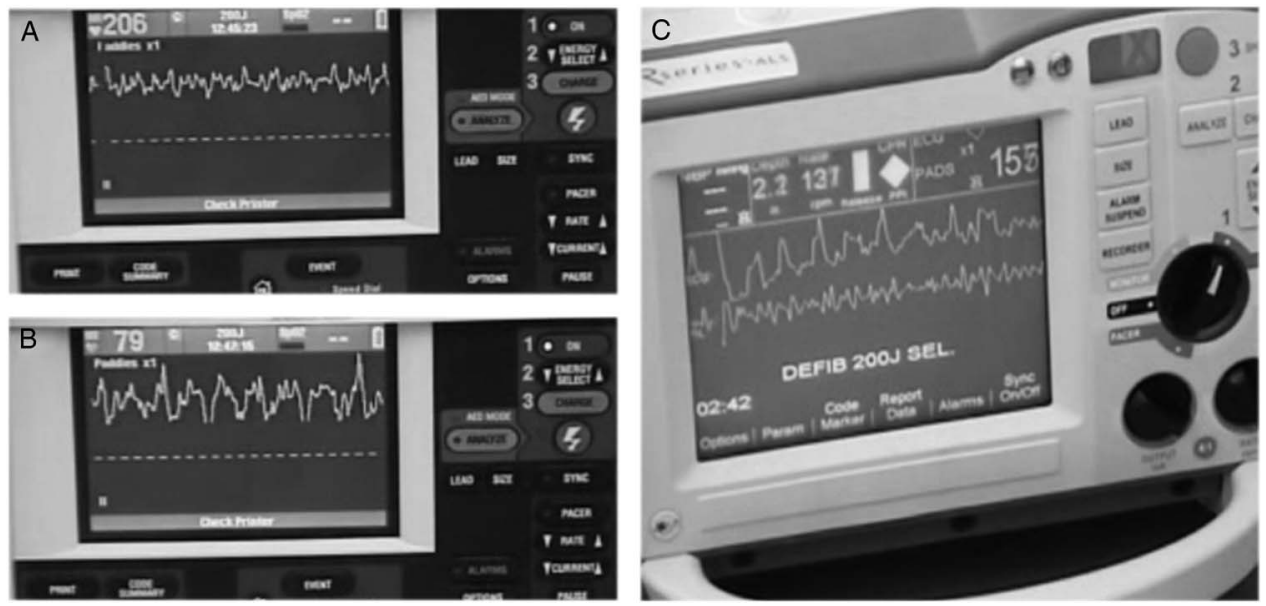

Figure 1. Comparing standard defibrillator to defibrillator equipped with AFT technology. A) On a standard defibrillator, when there are no ongoing compressions, the rhythm can successfully be read, as in the case of ventricular fibrillation. B) However, when there are ongoing compressions, artefact is seen in the trace, and the underlying rhythm cannot be reliably deduced. This necessitates a pause in compressions to check the underlying rhythm before shock is delivered. C) The AFT defibrillator demonstrates two traces during compressions. The top trace is the ECG trace with the artefact from compressions. The bottom trace is the filtered trace, with the artefact of compressions removed. In this case, the filtered rhythm is VF. Therefore, rescuers can analyse the underlying rhythm during compressions, eliminating the pause in compressions for rhythm analysis. AFT = artefact filtering technology; ECG = electrocardiogram; VF = ventricular fibrillation. 
improving $\mathrm{CPR}$, but the risks and benefits of this modification have not been well quantified.

The purpose of this simulation study was to isolate and evaluate the impact of AFT on CCF and peri-shock pause duration, as well as whether there was any additional effect of hands-on defibrillation on these parameters.

\section{METHODS}

This study was approved by the Queen's University Health Sciences Research Ethics Board.

\section{Setting and participants}

The study was conducted in the Kingston Resuscitation Institute's simulation lab at Queen's University. The manikin employed was the SimMan 3G (Laerdal Medical). To minimize variation and learning effect, we recruited a resuscitation team who had recently competed together in a simulation competition. Made up of three emergency medicine residents and an emergency department registered nurse, the team had knowledge and experience in CPR and cardiac arrest algorithms. Each rescuer maintained his or her same role (i.e., team leader, airway, and so forth) through all of the trials. Rescuers were blinded to the purpose of the study.

\section{Trial design}

Six trials of each experimental arm were run, with 18 performed in all. The order of the trials was determined by random number assignment.

The presenting rhythm of the scenarios was consistently ventricular fibrillation (VF) or pulseless ventricular tachycardia (VT) and proceeded in this rhythm until three shocks had been delivered. At that point, the rhythm was randomly changed to one of three possibilities: 1) asystole; 2) pulseless electrical activity (PEA); or 3) remaining in VF/VT. The simulation would then continue in this rhythm for the following 5 minutes. The participants were not informed of this pattern, in order to reduce learning effect. Each experimental arm consisted of the same number of asystole, PEA, and VF/VT trials.

There was a 5-minute rest period between trials to minimize fatigue. Participants used a 30:2 ratio of compressions to ventilations by a bag valve mask. Compressions were paused while breaths were delivered.
Participants performing compressions changed every five cycles. Rhythm analysis and defibrillation, when indicated, were completed every five cycles.

After completion of the study, all of the participants completed a brief questionnaire. They were simply asked to comment on the use of each protocol (see the following paragraph for experimental arm descriptions), with regards to comfort, ease of learning, and potential barriers to implementation. Participants were also asked to indicate any adverse effects that they may have noted. Questionnaires were completed by all participants during the debriefing period.

\section{Interventions}

There were three experimental arms used in this study, as follows:

1) Standard of Care: As per the existing ACLS algorithm for shockable arrest, participants paused compressions for both rhythm analysis and for delivery of shocks.

2) AFT: Participants used AFT to identify the underlying rhythm during compressions. Therefore, there was no pause for rhythm analysis, but participants did pause for shock delivery. Rescuers performed a brief confirmation check just prior to shock delivery, in order to ensure that a shockable rhythm continued to exist.

3) AFT with hands-on defibrillation: Rescuers used AFT to identify the underlying rhythm but continued compressions during shock delivery. There was a brief confirmation check just prior to shock delivery. Again, this was to ensure that a shockable rhythm continued to exist.

\section{Outcomes and analysis}

Each trial was recorded using simulation lab cameras and microphones. Video was reviewed, and the following times were measured: total length of resuscitation, total time that compressions were performed, pre-shock pause, and post shock pause. These times were then used to calculate CCF and peri-shock pause. Means for each of the three protocols were statistically analysed using the independent student's t-test, with a $p$ value of $<0.05$ considered significant.

\section{RESULTS}

CCF (Figure 2) was highest using AFT with hands-on defibrillation $(86.4 \pm 0.35 \%)$, as compared to $\mathrm{AFT}$ 
alone $(83.8 \pm 0.64 \% ; p<0.01)$. AFT provided a significant increase in CCF compared to the Standard of Care protocol $(76.7 \pm 0.72 \% ; p<0.001)$. Similarly, when looking at the peri-shock pause (Figure 3A), AFT with hands-on defibrillation also had the lowest perishock pause ( $2.6 \pm 0.35$ seconds) as compared to AFT alone $(5.3 \pm 0.64$ seconds, $p<0.001)$. AFT decreased the

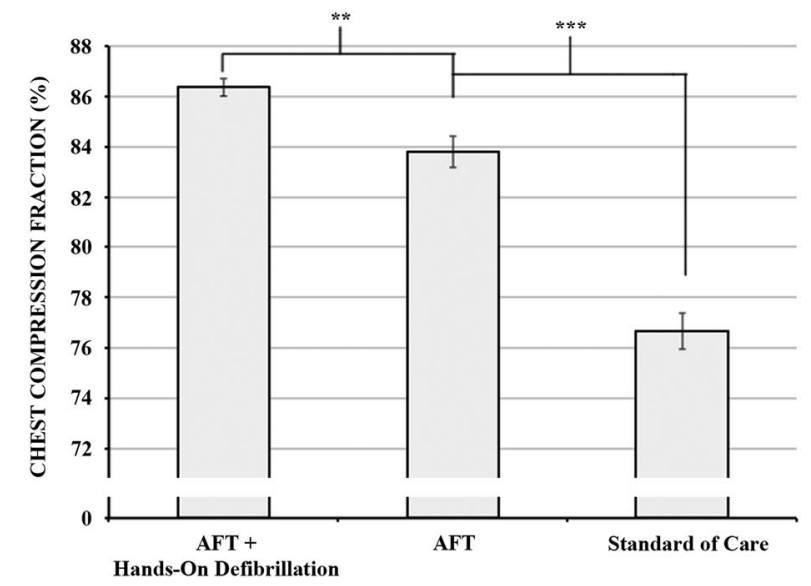

Figure 2. Both AFT and hands-on defibrillation increase the proportion of time spent doing chest compressions during shockable arrest. AFT with hands-on defibrillation was found to have the highest CCF, but AFT alone was still more efficient than our control protocol $\left(^{* *}=p<0.01\right.$; $\left.*^{* *}=p<0.001\right)$. AFT $=$ artefact filtering technology; $\mathrm{CCF}=$ chest compression fraction. peri-shock pause when compared to the Standard of Care $(7.4 \pm 0.22$ seconds). There were no differences between groups with regards to post-shock pause, suggesting that the improvement in peri-shock pause largely reflects reduced pre-shock pause (Figure 3, B and $\mathrm{C}$ ).

The rescuers did not report any difficulties in interpreting the underlying rhythm through the use of AFT, and there were no instances where a defibrillatory shock was delivered to a non-shockable rhythm. In the group employing AFT without hands-on defibrillation, no shocks were delivered while CPR was ongoing. As mentioned, all of the participants completed a questionnaire at study completion, where they were asked to comment on their comfort in the use of each of the protocols. The participants stated no obvious concerns regarding user comfort with AFT or hands-on defibrillation. Participants did note that potential safety concerns might limit the use of these modalities in real-world applications. There were no adverse effects noted across the study, although the safety of hands-on defibrillation was not a focus of this study.

\section{DISCUSSION}

Our study demonstrates that the use of AFT significantly improves CCF by reducing peri-shock

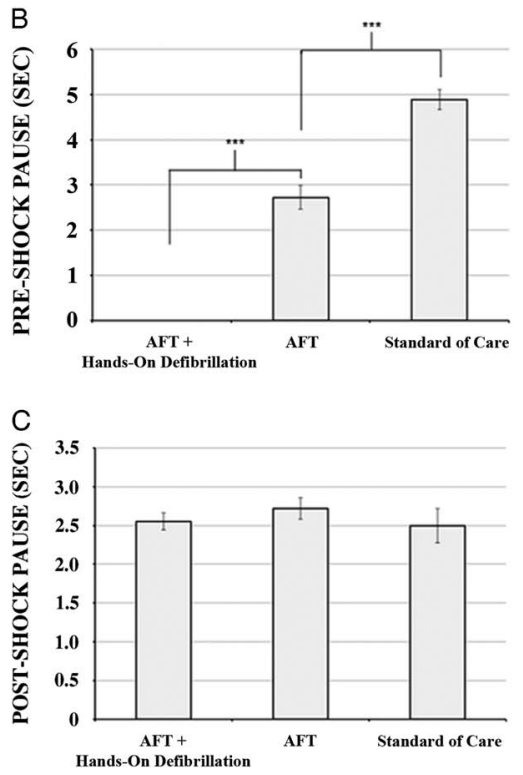

Figure 3. Shock pause duration between protocols. Peri-shock pause is the composite of pre-shock pause and post-shock pause. A) As can be seen, the use of AFT with hands-on defibrillation resulted in the lowest peri-shock pause. However, the use of AFT on its own was able to reduce peri-shock pause, as compared to the control $(* * *=p<0.001)$. B) These differences largely reflect a reduction in the pre-shock pause, because $C$ ) there were no significant differences between groups with regard to post-shock pause. AFT = artefact filtering technology. 
pause duration in shockable arrest. The reduction is due to a shortened pre-shock pause. We also demonstrated a small, but statistically significant, improvement in CCF with the addition of hands-on defibrillation.

Our work supports the existing work in this field. Barash et al. demonstrated as much as an $80 \%$ reduction in pre-shock pause time through the use of AFT. ${ }^{9}$ Our study did not find as substantial a reduction, possibly because we enrolled an expert group of participants and strictly adhered to ACLS recommendations to perform compressions during defibrillator charging. In a previous manikin study using a group of less-expert physicians, we found that the magnitude of improvement using AFT was much higher. ${ }^{12}$ The impact of this technology seems to be greatest in novice rescuers but is still evident in experienced hands.

To date, there has not been much work on real-life application of AFT in cardiac arrest. ${ }^{13}$ A study by Ruiz et al. found that AFT reduces pauses in chest compressions during shockable arrest ${ }^{14}$ and that the use of this particular AFT filter has reportedly high sensitivity $(91.0 \%)$ and specificity (96.6\%) for rhythm analysis during CPR in both shockable and non-shockable rhythms. ${ }^{15}$ Despite the encouraging numbers, most manufacturers recommend a confirmatory pause prior to shock delivery. For this reason, we incorporated this pause into our study, but, as confidence in the technology increases, further gains may be made in improving CCF.

Our results lend support to the hypothesis that hands-on defibrillation shortens shock pause duration and improves CCF. Although statistically significant, the improvement was small and may not be clinically significant enough to warrant the culture change that would be required for this therapy to be adopted.

\section{LIMITATIONS}

We expect that the improvement in CCF will result in an overall improvement in the effectiveness of CPR, but we did not measure the compression depth, rate of compression, or recoil. It is possible that there could be an unanticipated change in one of these variables that could mitigate the improvements. In addition, our experimental design did not include a "Hands-On Defibrillation Only" group. This was done purposely, because we felt as though the quantitative benefits of hands-on defibrillation on CPR quality had been previously demonstrated ${ }^{11}$ and were cognitively intuitive, as compared to the Standard of Care. Therefore, our objective was to investigate hands-on defibrillation as an adjunctive tool to AFT, in order to see whether the use of AFT negated any benefits of hands-on defibrillation (an important determination, given the perceived safety concerns of hands-on defibrillation). However, this meant that we were unable to compare hands-on defibrillation alone against AFT alone, with regards to our variables of interest.

Concerns have been raised regarding the safety of hands-on defibrillation. ${ }^{16,17}$ Because this was a simulation study, safety concerns related to hands-on defibrillation were minimal. The skin of the manikin does not conduct electricity, so we cannot make any conclusions from our results about the safety of the practice. It can be hypothesized that participants in this study felt comfortable using hands-on defibrillation because they knew there were no safety concerns. In a real-life application, CPR using hands-on defibrillation may be hindered by these concerns. Further research is required to address the balance of patient benefit and rescuer safety. Informed recommendations cannot be made about this practice until both are well quantified.

The underlying rhythm was easily discernible throughout our experiments, but there have been anecdotal reports in the prehospital setting of difficulties reading the rhythm from AFT during compressions. It is possible that our use of AFT in the simulation lab may better model the hospital environment.

Finally, our study focused on novel technology to reduce pre-shock pause and improve CCF during cardiac arrest. We are aware of other techniques, such as a "pit crew" concept ${ }^{18}$ and pre-charging of the defibrillator that may as well yield improvements in pre-shock pause and CPR fraction.

\section{CONCLUSION}

This study demonstrates that, in the simulation environment, AFT can provide significant improvement in CCF through a reduction in peri-shock pause, even in the hands of experienced rescuers. Hands-on defibrillation appears to offer additional improvement over AFT alone, suggesting that efforts to demonstrate its safety in the clinical setting are worthwhile.

Acknowledgements: We would like to thank Dr. Steve Brooks (Queen's University, Kingston, ON) for early comments on the manuscript and for his feedback during the process. We also 
thank Loren Fleming and Andy Howes for technical support. Zoll Medical Corporation provided us with an R-series defibrillator on loan, for completion of the study.

Competing interests: None declared.

\section{REFERENCES}

1. Cunningham LM, Mattu A, O'Connor RE, et al. Cardiopulmonary resuscitation for cardiac arrest: the importance of uninterrupted chest compressions in cardiac arrest resuscitation. Am 7 Emerg Med 2012;30(8):1630-8; doi:10.1016/j. ajem.2012.02.015.

2. Berg RA, Sanders AB, Kern KB, et al. Adverse hemodynamic effects of interrupting chest compressions for rescue breathing during cardiopulmonary resuscitation for ventricular fibrillation cardiac arrest. Circulation 2001;104:2465-70.

3. Field JM, Hazinski MF, Sayre MR, et al. 2010 American Heart Association guidelines for cardiopulmonary resuscitation and emergency cardiovascular care. Circulation 2010;122: S640-56; doi:10.1161/CIRCULATIONAHA.110.970889.

4. Paradis NA, Martin GB, Rivers EP, et al. Coronary perfusion pressure and the return of spontaneous circulation in human cardiopulmonary resuscitation. FAMA 1990;263(8):1106-13.

5. Christenson J, Andrusiek D, Everson-Stewart S, et al. Chest compression fraction determines survival in patients with out-of-hospital ventricular fibrillation. Circulation 2009;128:417-35; doi:10.1161/CIRCULATIONAHA.109. 852202.

6. Cheskes S, Schmicker RH, Christenson J, et al. Peri-shock pause: an independent predictor of survival from out-ofhospital shockable cardiac arrest. Circulation 2011;124:58-66; doi:10.1161/CIRCULATIONAHA.110.010736.

7. Cheskes S, Schmicker RH, Verbeek PR, et al. The impact of peri-shock pause on survival from out-of-hospital shockable cardiac arrest during the Resuscitation Outcomes Consortium (ROC) PRIMED trial. Resuscitation 2014;85(3): 336-42; doi:10.1016/j.resuscitation.2013.10.014.

8. Tan Q, Freeman G, Geheb F, et al. Electrocardiographic analysis during uninterrupted cardiopulmonary resuscitation. Crit Care Med 2008;36(11):S409-12.
9. Barash DM, Raymond RP, Tan Q, et al. A new defibrillator mode to reduce chest compression interruptions for health care professionals and lay rescuers: a pilot study in manikins. Prehosp Emerg Care 2011;15(1):88-97; doi:10.3109/ 10903127.2010.531375.

10. Kerber RE. Gloves to protect hands-and heart. Resuscitation 2013;84:859-60; doi:10.1016/j.resuscitation.2013.04.013.

11. Neumann T, Gruenewald M, Lauenstein C, et al. Hands-on defibrillation has the potential to improve the quality of cardiopulmonary resuscitation and is safe for rescuers-a preclinical study. 7 Am Heart Assoc 2012;1(5):e001313, doi:10.1161/JAHA.112.001313.

12. Fernando SM, Baylis JB, Szulewski A, et al. Motion artifact reduction of ECG signal allows for greater chest compression fraction during CPR. CFEM 2013;15(Suppl 1):Available at: http://caep.ca/sites/caep.ca/files/caep/CAEP2013/Presentations/ shannon_fernando_-_caep_poster_widescreen_version_1.pdf.

13. Ruiz J, Ayala U, Ruiz de Gauna S, et al. Direct evaluation of the effect of filtering the chest compression artifacts on the uninterrupted cardiopulmonary resuscitation time. $\mathrm{Am} \mathcal{7}$ Emerg Med 2013;31(6):910-5; doi:10.1016/j.ajem.2013. 02.044 .

14. Ruiz de Gauna S, Irusta U, Ruiz J, et al. Rhythm analysis during cardiopulmonary resuscitation: past, present, and future. Biomed Res Int 2014;2014:386010, doi:10.1155/ 2014/386010.

15. Ayala $U$, Irusta $U$, Ruiz $J$, et al. A reliable method for rhythm analysis during cardiopulmonary resuscitation. Biomed Res Int 2014;2014:872470, doi:10.1155/2014/872470.

16. Lemkin DL, Witting MD, Allison MG, et al. Electrical exposure risk associated with hands-on defibrillation. Resuscitation 2014;85(10):1330-6; doi:10.1016/j.resuscitation. 2014.06.023.

17. Thomson JE, Petley GW, Deakin CD. Risk of injury to rescuers who use hands-on defibrillation. Resuscitation 2013;84:e131-2; doi:10.1016/j.resuscitation.2013.04.031.

18. Ong ME, Quah JL, Annathurai A. Improving the quality of cardiopulmonary resuscitation by training dedicated cardiac arrest teams incorporating a mechanical load-distributing device at the emergency department. Resuscitation 2013;84:508-14; doi:10.1016/j.resuscitation.2012.07.033. 